\title{
Self-phase modulation of a single-cycle terahertz pulse by nonlinear free-carrier response in a semiconductor
}

Turchinovich, Dmitry; Hvam, Jørn Märcher; Hoffmann, Matthias C.

Published in:

Physical Review B Condensed Matter

Link to article, DOI:

10.1103/PhysRevB.85.201304

Publication date:

2012

Document Version

Publisher's PDF, also known as Version of record

Link back to DTU Orbit

Citation (APA):

Turchinovich, D., Hvam, J. M., \& Hoffmann, M. C. (2012). Self-phase modulation of a single-cycle terahertz pulse by nonlinear free-carrier response in a semiconductor. Physical Review B Condensed Matter, 85(20), 201304. https://doi.org/10.1103/PhysRevB.85.201304

\section{General rights}

Copyright and moral rights for the publications made accessible in the public portal are retained by the authors and/or other copyright owners and it is a condition of accessing publications that users recognise and abide by the legal requirements associated with these rights.

- Users may download and print one copy of any publication from the public portal for the purpose of private study or research.

- You may not further distribute the material or use it for any profit-making activity or commercial gain

- You may freely distribute the URL identifying the publication in the public portal 


\title{
Self-phase modulation of a single-cycle terahertz pulse by nonlinear free-carrier response in a semiconductor
}

\author{
Dmitry Turchinovich, ${ }^{1,2, *}$ Jørn M. Hvam, ${ }^{1}$ and Matthias C. Hoffmann ${ }^{3}$ \\ ${ }^{1}$ DTU Fotonik, Technical University of Denmark, DK-2800 Kgs. Lyngby, Denmark \\ ${ }^{2}$ Max Planck Institute for Polymer Research, Ackermannweg 10, 55128 Mainz, Germany \\ ${ }^{3}$ SLAC Linear Accelerator Laboratory, 2575 Sand Hill Road, Menlo Park, California, 94025, USA
}

(Received 23 January 2012; published 29 May 2012)

\begin{abstract}
We investigate the self-phase modulation (SPM) of a single-cycle terahertz pulse in a semiconductor, using bulk $n$-GaAs as a model system. The SPM arises from the heating of free electrons in the electric field of the terahertz pulse, leading to an ultrafast reduction of the plasma frequency, and hence to a strong modification of the terahertz-range dielectric function of the material. Terahertz SPM is observed directly in the time domain. In the frequency domain it corresponds to a strong frequency-dependent refractive index nonlinearity of $n$-GaAs, found to be both positive and negative within the broad spectrum of the terahertz pulse, with the zero-nonlinearity point defined by the electron momentum relaxation rate. We also observed the nonlinear spectral broadening and compression of the terahertz pulse.
\end{abstract}

DOI: 10.1103/PhysRevB.85.201304

PACS number(s): 78.20.-e, 42.65.Re, 72.20.Ht, 78.47.J-

Since its first demonstration ${ }^{1}$ more than five decades ago, nonlinear optics (NLO) has revolutionized the field of photonics. Until recently, ultrafast NLO was largely confined to the visible and infrared spectral ranges. However, with the emergence of sources generating high-energy ultrafast singlecycle terahertz $(\mathrm{THz})$ pulses, ${ }^{2-4}$ more and more NLO studies are being conducted in the $\mathrm{THz}$ range. Nonlinear dynamics in semiconductors in strong $\mathrm{THz}$ fields is a particularly rich area of research due to good coupling between the $\mathrm{THz}$ fields and free carriers, and due to the possibility of inducing strong polarization effects in semiconductors with strong $\mathrm{THz}$ signals. ${ }^{5}$ Many interesting ultrafast phenomena have been investigated recently, such as interaction of strong $\mathrm{THz}$ fields with excitons, ${ }^{6,7} \mathrm{THz}$-induced impact ionization of electrons in narrow-gap semiconductors, ${ }^{8,9}$ ultrafast THzinduced electron dynamics in complex conduction bands, ${ }^{10-14}$ and femtosecond all-optical switching in nanoscale structures with THz signals. ${ }^{15,16}$

The majority of studies in the present-day ultrafast $\mathrm{THz}$ NLO are focused on achieving a better understanding and control of nonlinear processes in (mostly semiconductor) materials using THz light. There are, however, very few reports aiming at control and manipulation of $\mathrm{THz}$ light making use of the material's nonlinear response. Yet, the ultrafast $\mathrm{THz}$ signals are usually single-cycle wave forms, which can be experimentally sampled with a time resolution much better than one optical cycle, providing the complete time-domain information about the optical signal-its amplitude and phase. ${ }^{17,18}$ As we will show in this Rapid Communication, this makes the $\mathrm{THz}$ pulses ideal tools for the direct observation of both (i) the THz-specific NLO phenomena and (ii) the general NLO phenomena occurring in the single-cycle optical regime.

Single-cycle pulses can not be described by the traditional carrier-envelope approximation. ${ }^{19}$ Irrespective of the frequency range to which they belong, they inherently have an extremely broad spectral bandwidth covering many octaves of frequencies. Hence the propagation of such signals in the medium is governed by the complex-valued material dielectric function $\hat{\varepsilon}(\omega)$ covering a very broad spectral range.
An optical nonlinearity - the near-instantaneous modification of this dielectric function due to a nonlinear light-matter interaction occurring in the single-cycle regime, corresponds to a modification of the optical properties of matter over an extremely broad range of frequencies. We find the effect of such a sudden change of dielectric environment on the propagating single-cycle wave form to be quite intriguing, which is the motivation behind the present work.

In this Rapid Communication, we report on the study of a fundamental nonlinear optical effect-the self phase modulation (SPM) $)^{20,21}$ of a single-cycle THz pulse. The SPM occurs due to the change of the (real part of) refractive index $n(\omega)=\operatorname{Re} \sqrt{\hat{\varepsilon}(\omega)}$ of the material as a result of light-matter interaction. As an efficient nonlinear medium for the $\mathrm{THz}$ range we used an $n$-doped bulk semiconductor GaAs, whose optical nonlinearity arises from the response of free electrons to the THz fields. We note that any other doped or photoexcited semiconductor with a complex band structure will also exhibit the THz-range SPM according to the mechanism described here. The SPM was observed by us directly in the time domain using THz time-domain spectroscopy (TDS) ${ }^{17,18}$ and was analyzed in the frequency domain.

In this Rapid Communication, we show that the THz-range optical nonlinearity of a doped or photoexcited semiconductor is based on the modification of its plasma frequency by the carrier heating in the $\mathrm{THz}$ field. Semiconductors with free carriers are good absorbers of $\mathrm{THz}$ radiation, and their THz-range complex-valued dielectric function $\hat{\varepsilon}(\omega)$ is well described by the basic Drude plasma model ${ }^{22,23}$

$$
\hat{\varepsilon}(\omega)=(n+i \alpha c / 2 \omega)^{2}=\varepsilon_{d c}-\omega_{p}^{2} /\left(\omega^{2}-i \omega / \tau_{r}\right)
$$

or by one of its extensions. ${ }^{24,25}$ Here, $n$ and $\alpha$ are the frequency-dependent refractive index and power absorption coefficient, respectively, $\tau_{r}$ is a carrier momentum relaxation time, and $\varepsilon_{\mathrm{dc}}$ is the background "static" dielectric constant of the semiconductor in the absence of free carriers. ${ }^{26}$ $\omega_{p}=\left(N e^{2} / \epsilon_{0} m\right)^{1 / 2}$ is the plasma frequency, where $e$ is the elementary charge, $N$ is the free-carrier density, $\epsilon_{0}$ is the vacuum permittivity, and $m$ is the carrier effective mass. 
It directly follows from Eq. (1) that the change in plasma frequency $\omega_{p}$ resulting from the THz light-matter interaction will lead to a change in the $\mathrm{THz}$ absorption $\alpha$ and the refractive index $n$ of the semiconductor, i.e., to nonlinear absorption and SPM. In the limiting case of $\omega_{p} \rightarrow 0$ the $\mathrm{THz}$ absorption will vanish, and the $\mathrm{THz}$ refractive index will be defined by the background dielectric constant $n\left(\omega_{p} \rightarrow 0\right)=\sqrt{\varepsilon_{\mathrm{dc}}}$. The only mechanism to reduce the plasma frequency in a doped semiconductor (i.e., where $N$ is fixed) is the increase of the carrier effective mass $m$. In $n$-doped materials (e.g., $n$-GaAs), this can be achieved by electron heating in a sufficiently strong electric field by redistribution of the electron population in energy-momentum space of the conduction band from lowmomentum, small-effective-mass states around the bottom of the $\Gamma$ valley to high-momentum, large-effective mass states in the satellite valleys and to the states of strong nonparabolicity within the $\Gamma$ valley (see, e.g., Ref. 27 and references therein). In $p$-doped materials, the hole scattering between the hole subbands can be utilized.

Recently, the carrier heating in the ponderomotive potential created by the electric field of a single-cycle $\mathrm{THz}$ pulse $\mathrm{e}^{10-14}$ was demonstrated. In Ref. 10, it was shown that right after the excitation of $n$-GaAs by a THz pulse with peak electric field $E_{\mathrm{THz}} \simeq 100 \mathrm{kV} / \mathrm{cm}$, the effective mass of electrons initially occupying the bottom of $\Gamma$ valley $\left(m_{\Gamma}=0.063 m_{0}\right)$ drastically increases and reaches the values of $m \simeq 0.3 m_{0}$, more typical for the $L$ and $X$ valley states in GaAs $\left(m_{L}=0.23 m_{0}\right.$, and $\left.m_{X}=0.43 m_{0}\right){ }^{27,28}$ Here $m_{0}$ is the electron rest mass. The $\mathrm{THz}$ saturable absorption based on this mechanism, shown in Fig. 1(a), was observed by us in various $n$-doped semiconductors. ${ }^{29}$ The temporal response of $\mathrm{THz}$ nonlinearity of $n$-GaAs is near instantaneous, as shown in Fig. 1(b). This measurement is a result of $\mathrm{THz}$ pump- $\mathrm{THz}$ probe (TPTP) spectroscopy ${ }^{10,12-14}$ where our $n$-GaAs sample with the thickness $d=0.4 \mathrm{~mm}, N=5 \times 10^{15} \mathrm{~cm}^{-3}$, and $\tau_{r}=94$ fs was excited by a $\mathrm{THz}$ pump pulse with peak $E_{\mathrm{THz}} \simeq 200$ $\mathrm{kV} / \mathrm{cm}$. The near-instantaneous bleaching of the sample is followed by a slower recovery as the electrons return to the bottom of the $\Gamma$ valley. ${ }^{30}$ This and all other measurements in this work were performed at room temperature.

We studied the SPM of single-cycle $\mathrm{THz}$ pulses in our $n$-GaAs sample in a nonlinear THz TDS experiment set up in transmission configuration. ${ }^{29}$ The frequency spectrum of the $\mathrm{THz}$ pulses, generated by optimized optical rectification of $800 \mathrm{~nm}, 100$-fs laser pulses in lithium niobate (LN), ${ }^{2-4}$ covered the range $0.1-3 \mathrm{THz}$, thus containing many octaves of frequencies. The peak electric field $E_{\mathrm{THz}}$ in the generated $\mathrm{THz}$ pulses was adjusted in the range of 9-292 kV/cm using a pair of wire-grid polarizers positioned after the $\mathrm{THz}$ emitter in the spectrometer. ${ }^{29} \mathrm{THz}$ transmission through the polarizers is nominally frequency independent. The $\mathrm{THz}$ pulses were recorded in the time domain using free-space electro-optic sampling. ${ }^{17,18}$

As shown in Fig. 2, for each value of peak $E_{\mathrm{THz}}$ in the generated $\mathrm{THz}$ pulse, a reference (travelling through the optical path in the spectrometer in vacuum) and a sample (travelling through the same geometrical path, but with the sample inserted into it) $\mathrm{THz}$ pulses, ${ }^{17,18}$ centered around the time delays of 0 and $3.3 \mathrm{ps}$, respectively, were recorded. A clear $\mathrm{THz}$ saturable absorption (i.e., increase in $\mathrm{THz}$ transmission) ${ }^{29}$ in our $n$-GaAs sample with growth in peak electric field $E_{\mathrm{THz}}$ is observed. In Figs. 2(a) and 2(c), the measured reference and sample THz pulses for the two extreme values of peak $E_{\mathrm{THz}}$, 292 and $9 \mathrm{kV} / \mathrm{cm}$, are shown as examples of raw data. Here and below, all mentioned peak values of $E_{\mathrm{THz}}$ correspond to that of the reference $\mathrm{THz}$ pulse and are reported as measured at the position of the sample.

In Fig. 2(b), a two-dimensional plot shows the reference and sample pulses normalized to their respective peak values, for the whole range of peak $E_{\mathrm{THz}}$ in our experiments. As apparent from this plot, the time delay between the reference and the sample $\mathrm{THz}$ pulses in $n$-GaAs increases with increasing peak $E_{\mathrm{THz}}$, which is a direct signature of SPM. The dashed black line in Fig. 2(b) shows the estimated group delay (i.e., "delay as a whole" $)^{29}$ of a $\mathrm{THz}$ pulse in the material as a function of peak $E_{\mathrm{THz}}$. In Figs. 2(d) and 2(e), the normalized reference and sample pulses for the two extreme values of peak $E_{\mathrm{THz}}$ of 9 and $292 \mathrm{kV} / \mathrm{cm}$ are shown. The difference between the two reference wave forms in Fig. 2(d) is fairly small: there is no observable time shift, and there is only a very small change in the pulse shapes, which is due to nonideality of the $\mathrm{THz}$ wire-grid polarizers used for adjustment of the peak $\mathrm{THz}$ field. The difference between the two sample wave forms in Fig. 2(e) is, however, quite noticeable. The sample wave form corresponding to a stronger $E_{\mathrm{THz}}$ clearly experiences a larger time delay as well as a certain reshaping. Figure 2 is therefore the direct time-domain observation of $\mathrm{THz}$ SPM occurring in a single-cycle regime. In Fig. 2(f), we show
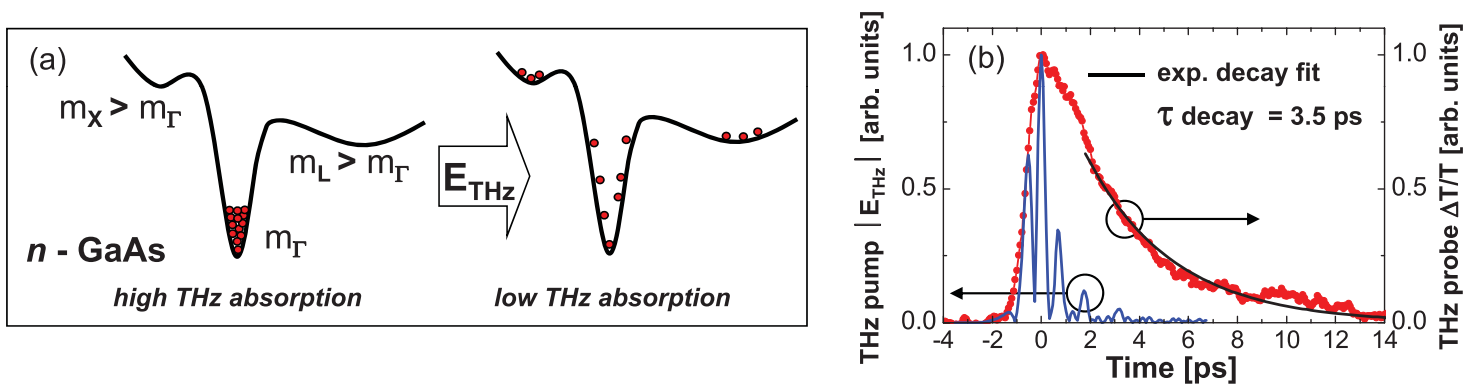

FIG. 1. (Color online) (a) Mechanism of THz nonlinearity in $n$-GaAs, based on carrier heating in the electric field of a THz pulse, leading to a decrease of plasma frequency $\omega_{p}$ and saturation of THz absorption. (b) Temporal dynamics of THz nonlinearity in $n$-GaAs, observed in a transmission-mode TPTP measurement. Near-instantaneous bleaching of the sample via carrier-heating in the THz field is followed by a slower recovery due to electron cooling. 

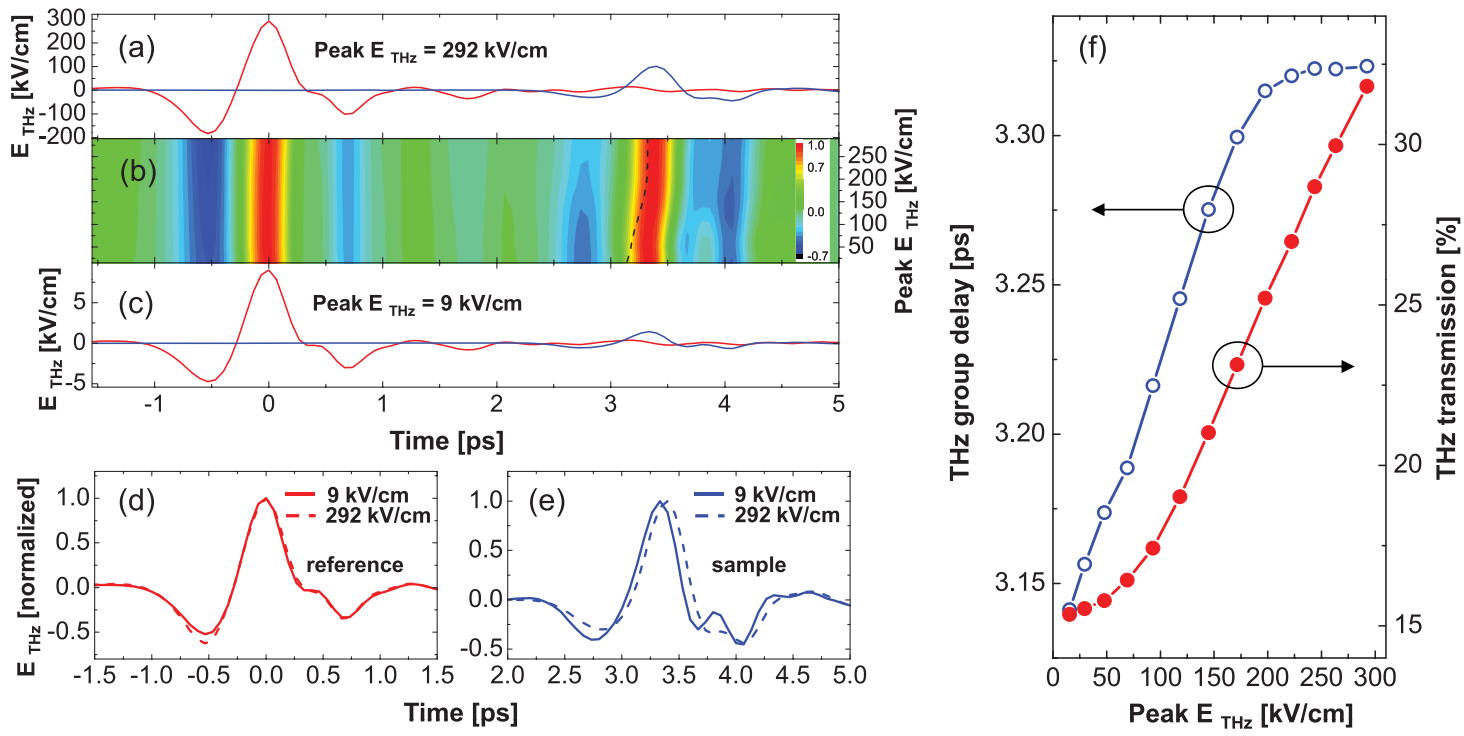

FIG. 2. (Color online) THz SPM in $n$-GaAs in the time domain. Peak $E_{\mathrm{THz}}$ of reference THz pulse in (a) is $292 \mathrm{kV} / \mathrm{cm}$, and in (c) is $9 \mathrm{kV} / \mathrm{cm}$. (b) Reference and sample pulses normalized to their maxima, for the whole range of peak $E_{\mathrm{THz}}$ of $9-292 \mathrm{kV} / \mathrm{cm}$. Black dashed line: estimated group delay of the sample pulse. Normalized reference (d) and sample (e) pulses for the cases of peak $E_{\mathrm{THz}}$ of 9 and $292 \mathrm{kV} / \mathrm{cm}$, demonstrating the SPM in the sample. (f) Amplitude frequency-integrated transmission coefficient, and estimated group delay of a sample pulse as a function of peak $E_{\mathrm{THz}}$.

the connection between the saturable absorption and SPM in our sample by plotting both the $\mathrm{THz}$ frequency-integrated amplitude transmission coefficient and an estimated group delay of the sample pulse as a function of peak $E_{\mathrm{THz}}$. The observed increase in the transmission coefficient is more than twofold, and the increase in the group delay exceeds $150 \mathrm{fs}$, making it a noticeable fraction of the duration of the THz cycle.

We now transfer to the frequency domain by using the Fourier transforms of the measured $\mathrm{THz}$ pulses from Fig. 2. ${ }^{17,18}$ In Fig. 3, we show the SPM as a nonlinear, THz-field dependent contribution to the refractive index of $n$-GaAs, calculated as a correction to the refractive index spectrum measured at the weakest peak $E_{\mathrm{THz}}, \Delta n(\omega)=n\left(\omega, E_{\mathrm{THz}}\right)-$ $n(\omega, 9 \mathrm{kV} / \mathrm{cm})$. A very strong, and also frequency-dependent, dynamics of $\Delta n(\omega)$ is observed with increase in peak $E_{\mathrm{THz}}$ from 9 to $292 \mathrm{kV} / \mathrm{cm}$. In the inset, the examples of estimated ${ }^{31}$ absorption spectra are shown for the same range of peak $E_{\mathrm{THz}}$. Interestingly, the refractive index nonlinearity is found to be both negative and positive within the bandwidth of the same single-cycle $\mathrm{THz}$ pulse, making it quite a unique situation in nonlinear optics (though quite understandable given the ultra-broadband nature of single-cycle pulses). These negative and positive trends in the dynamics of $\Delta n$ at the frequencies below and above approximately $0.5 \mathrm{THz}$, with increase in peak $E_{\mathrm{THz}}$, are shown with blue arrows in Fig. 3. The maximum refractive index changes in our measurements were as large as $\Delta n=-0.13$ to +0.08 , in the spectral range $0.3-2.4 \mathrm{THz}$. This corresponds to the nonlinear phase delay $\Delta \tau_{\phi}=\Delta n d / c$ in the range of -173 to $+107 \mathrm{fs}$, acquired by the $\mathrm{THz}$ signal at different frequencies in our 0.4-mm thick sample, as also shown in Fig. 3.

In our specific case, most of the spectrum of the $\mathrm{THz}$ pulse (shown as grey area in Fig. 3) was in the spectral range of positive index nonlinearity, which results in the increasing group delay of the $\mathrm{THz}$ pulse at higher peak $E_{\mathrm{THz}}$ (see Fig. 2). However, if the $\mathrm{THz}$ pulse spectrum belonged to the range below $0.5 \mathrm{THz}$, the decrease in group delay with increase in $E_{\mathrm{THz}}$ would occur instead.

Inherent to SPM is spectral reshaping of the optical signal, ${ }^{36,37}$ which can lead to both spectral broadening and compression within the same NLO system (see, e.g., Ref. 32). In Fig. 4(a), it is illustrated by the dependency of the ratio of effective spectral bandwidths of the sample and reference pulses $\Delta \omega_{\text {sam }} / \Delta \omega_{\text {ref }}$ on peak $E_{\mathrm{THz}} \cdot{ }^{33}$ If this ratio is

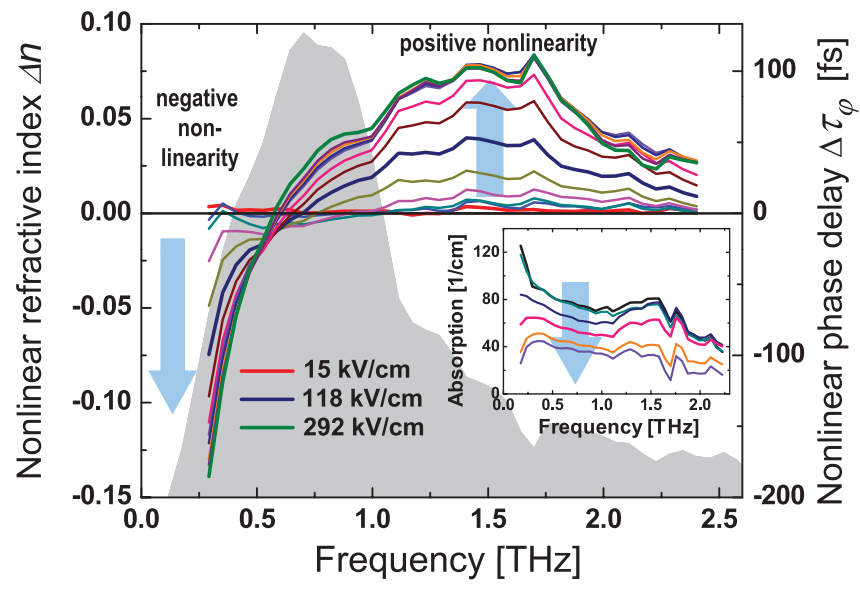

FIG. 3. (Color online) THz SPM in $n$-GaAs in spectral domain. Nonlinear contribution to refractive index $\Delta n$, and corresponding nonlinear phase delay as a function of frequency and peak $E_{\mathrm{THz}}$ in the range $9-292 \mathrm{kV} / \mathrm{cm}$. The amplitude spectrum of reference $\mathrm{THz}$ pulse is shown as a background. Inset: selected estimated absorption spectra in the same range of peak $E_{\mathrm{THz}}$. Blue arrows show the positive and negative refractive index nonlinearity, and decrease in absorption, with increasing peak $E_{\mathrm{THz}}$. 

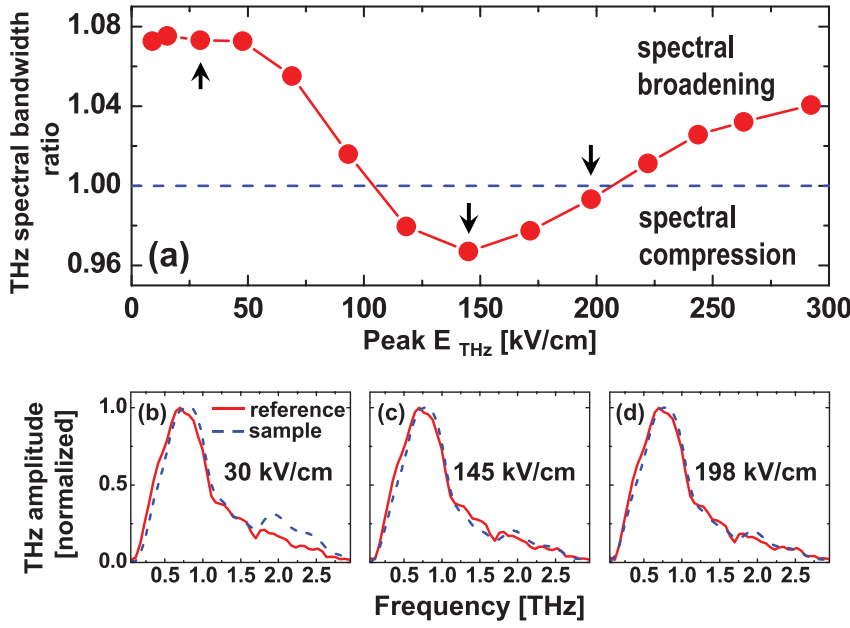

FIG. 4. (Color online) Nonlinear spectral broadening and compression of a THz pulse in $n$-GaAs. (a) The ratio of effective bandwidths of the sample and reference pulses, as a function of peak $E_{\mathrm{THz}}$. (b)-(d) Examples of spectral dynamics: normalized amplitude spectra of reference and sample $\mathrm{THz}$ pulses measured at peak $\mathrm{THz}$ field values of 30,145 , and $198 \mathrm{kV} / \mathrm{cm}$, indicated by arrows in (a).

greater (smaller) than unity, then relative spectral broadening (compression) of a $\mathrm{THz}$ pulse in a sample takes place. We observed a spectral compression and broadening of a $\mathrm{THz}$ pulse in our sample by approximately $-3 \%$ to $+7 \%$. In Figs. 4(b)-4(d), the normalized amplitude spectra of reference and sample $\mathrm{THz}$ pulses at selected values of peak $E_{\mathrm{THz}}$ are shown, exemplifying the spectral broadening, compression, and virtually no change, respectively, of THz pulses in $n$-GaAs.

In a movie (see Supplemental Material ${ }^{38}$ ) we visualize the correlation between the $\mathrm{THz}$ pulse transmission, delay, and spectral dynamics as a function of peak $E_{\mathrm{THz}}$, also showing the field-dependent evolution of $\mathrm{THz}$ pulse spectrograms ${ }^{34}$.

In general, the nonlinear-optical spectral dynamics will depend on the combination of pulse chirp, index nonlinearity, and frequency-dependent nonlinear absorption. ${ }^{36,37}$ As the $\mathrm{THz}$ SPM finds its origin in the ponderomotive acceleration of free electrons, the $\mathrm{THz}$ pulse shape at each moment during its propagation becomes an important factor in precise time-domain modeling of $\mathrm{THz}$ nonlinearities in semiconductors, which can only be achieved using advanced numerical methods. ${ }^{6,14}$

We note that the link between the refractive index and absorption (or gain) nonlinearities is fundamental, and is used, e.g., in semiconductor slow/fast light devices for the telecom range (see, e.g., Refs. 35 and 36). We also note that the reshaping of a $\mathrm{THz}$ signal generated in a $\mathrm{LN}$ crystal under variable optical pump strength was reported and assigned to $\mathrm{THz}$ SPM in Ref. 3, but later study clearly related this effect to the optical-range nonlinearity of the laser pump pulse in LN. ${ }^{39}$ The reshaping by SPM of a THz pulse in InSb undergoing impact ionization was reported, ${ }^{8}$ however, without the spectral-domain analysis.

Now we illustrate the observed trends in nonlinear absorption and refractive index by simple modeling. In Fig. 5, the measured linear $\mathrm{THz}$ spectra of our sample are shown (symbols) along with the Drude fit using the parameters

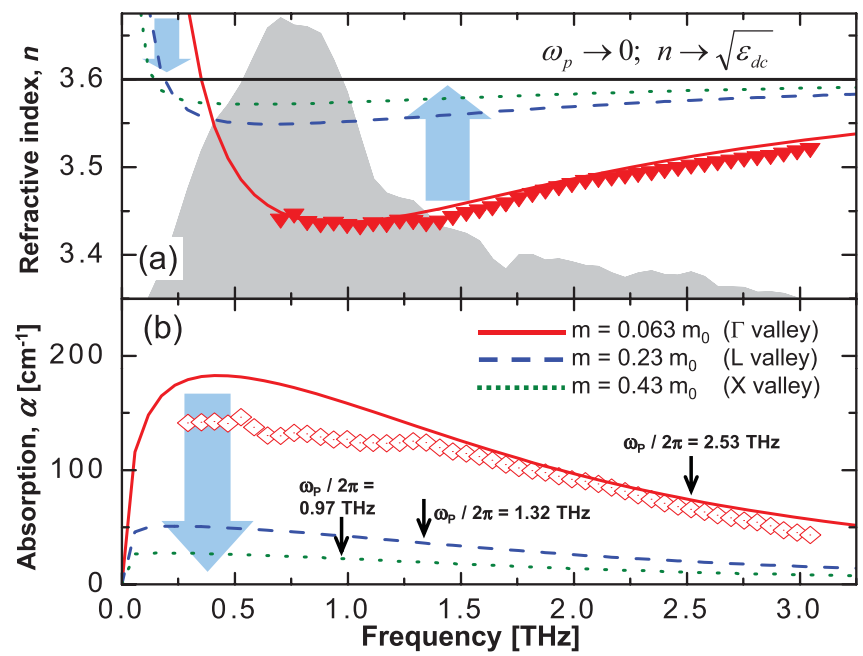

FIG. 5. (Color online) Blue arrows: trends in (a) refractive index and (b) absorption $\mathrm{THz}$ spectra of $n$-GaAs with decrease in $\omega_{p}$. Spectra measured in linear regime (symbols) and calculated using a DS model with $N=5 \times 10^{15} \mathrm{~cm}^{-3}, \tau_{r}=94 \mathrm{fs}$, and $c_{\mathrm{DS}}=-0.15$ for the electron effective mass of $\Gamma$ (red solid line), $L$ (blue dashed line), and $X$ (green dotted line) valleys. Amplitude spectrum of a reference $\mathrm{THz}$ pulse is shown in the background of (a). Black arrows indicate the corresponding $\omega_{p}$ values. Horizontal line in (a) corresponds to $\omega_{p}=0$, when $n=\sqrt{\varepsilon_{\mathrm{dc}}}=3.6$.

$N$ and $\tau_{r}$ described above, $n=\sqrt{\varepsilon_{\mathrm{dc}}}=3.6,{ }^{40}$ and $m=m_{\Gamma}$ (solid lines). For this fit, we used a Drude-Smith (DS) model incorporating the effective carrier localization into an ideal Drude model. ${ }^{24,25}$ The small localization parameter $c_{\mathrm{DS}}=$ -0.15 used here shows that our sample is very close to an ideal Drude plasma system described by Eq. (1). In the same figure, we show the calculated $n$ and $\alpha$ spectra for the same sample parameters as before, but using the heavier effective masses $m=m_{L}$ and $m=m_{X}$ of the satellite valleys ${ }^{28,42}$ (dashed and dotted lines, respectively). Corresponding values for $\omega_{p}$ are shown in the figure using black arrows. It is clear that with decrease in $\omega_{p}$ the absorption decreases monotonously. The trend in refractive index with decreasing $\omega_{p}$ is the decrease from larger values at lower frequencies $(<0.5 \mathrm{THz})$ and increase from lower values at higher frequencies, approaching the background value of $n=\sqrt{\varepsilon_{\mathrm{dc}}}$ at $\omega_{p}=0$. These trends in the dynamics of $\alpha$ and $n$, indicated by the blue arrows, exactly match the trends in observed saturable absorption and refractive index nonlinearity [see Figs. 2(f) and 3], including opposite signs of $\Delta n$ at lower and higher frequencies. The large value of refractive index at lower frequencies in a metal or doped semiconductor directly follows from Eq. (1): it is known as Hagen-Rubens regime, and is characteristic for the frequency range $\omega \ll \tau_{r}^{-1} .^{23}$ The spectral position of zero index nonlinearity is thus defined by the electron momentum relaxation rate $\tau_{r}^{-1}$, and can be theoretically predicted with reasonable accuracy, as shown by Figs. 3 and $5(\mathrm{a})$.

In conclusion, we have studied the SPM of a single-cycle $\mathrm{THz}$ pulse in a semiconductor, whose $\mathrm{THz}$-range optical nonlinearity is based on the carrier heating in $\mathrm{THz}$ fields leading to the plasma frequency shift, and can be adequately 
described by the Drude model. It can find its application in, e.g., THz-wave ultrahigh speed all-optical signal processing schemes, where THz SPM can be employed as successfully as in the optical domain, ${ }^{35-37}$ although the physical mechanisms of $\mathrm{THz}$ and optical range nonlinearities are very different.

Material engineering can be employed to minimize the effect of linear absorption on the propagation of $\mathrm{THz}$ pulses, as well as to control the electron momentum relaxation rate, which defines the sign of refractive index nonlinearity. Combination of smaller linear loss with engineered index nonlinearity will pave the way to further development of
$\mathrm{THz}$ nonlinear optics, and will enable $\mathrm{THz}$ supercontinuum generation and $\mathrm{THz}$ solitons in solid-state systems, which are presently only achievable in the optical range.

We are grateful to K. Yvind, J. Lægsgaard, J. Mørk (DTU Fotonik), and A. Cavalleri (University of Hamburg) for valuable discussions. We acknowledge the partial financial support from the Danish Proof of Concept Foundation (grant 7.7 Ultrahigh-speed wireless data communications), Danish Council for Independent Research - Technology and Production Sciences (FTP), and Max Planck Society. *dmtu@fotonik.dtu.dk

${ }^{1}$ P. A. Franken, A. E. Hill, C. W. Peters, and G. Weinreich, Phys. Rev. Lett. 7, 118 (1961).

${ }^{2}$ K.-L. Yeh, M. C. Hoffmann, J. Hebling, and K. A. Nelson, Appl. Phys. Lett. 90, 171121 (2007).

${ }^{3}$ J. Hebling, K.-L. Yeh, M. C. Hoffmann, and K. A. Nelson, J. Sel. Top. Quant. Electron. 14, 345 (2008).

${ }^{4}$ M. C. Hoffmann and J. A. Fulop, J. Phys. D 44, 083001 (2011).

${ }^{5}$ S. D. Ganichev and W. Prettl, Intense Terahertz Excitation of Semiconductors (Oxford University Press, Oxford, 2006).

${ }^{6}$ J. R. Danielson, Y.-S. Lee, J. P. Prineas, J. T. Steiner, M. Kira, and S. W. Koch, Phys. Rev. Lett. 99, 237401 (2007).

${ }^{7}$ H. Hirori, M. Nagai, and K. Tanaka, Phys. Rev. B 81, 081305 (2010).

${ }^{8}$ H. Wen, M. Wiczer, and A. M. Lindenberg, Phys. Rev. B 78, 125203 (2008).

${ }^{9}$ M. C. Hoffmann, J. Hebling, H. Hwang, K.-L. Yeh, and K. Nelson, Phys. Rev. B 79, 161201 (2009).

${ }^{10}$ M. C. Hoffmann, J. Hebling, H. Y. Hwang, K.-L. Yeh, and K. A. Nelson, J. Opt. Soc. Am. B 26, A29 (2009).

${ }^{11}$ F. H. Su et al., Opt. Express 17, 9620 (2009).

${ }^{12}$ J. Hebling, M. C. Hoffmann, H. Y. Hwang, K.-L. Yeh, and K. A. Nelson, Phys. Rev. B 81, 035201 (2010).

${ }^{13}$ G. Sharma et al., IEEE Photonics J.2, 578 (2010).

${ }^{14}$ F. Blanchard, D. Golde, F. H. Su, L. Razzari, G. Sharma, R. Morandotti, T. Ozaki, M. Reid, M. Kira, S. W. Koch, and F. A. Hegmann, Phys. Rev. Lett. 107, 107401 (2011).

${ }^{15}$ M. C. Hoffmann, B. S. Monozon, D. A. Livshits, E. U. Rafailov, and D. Turchinovich, Appl. Phys. Lett. 97, 231108 (2010).

${ }^{16}$ T. Ogawa, S. Watanabe, N. Minami, and R. Shimano, Appl. Phys. Lett. 97, 041111 (2010).

${ }^{17}$ M. Tonouchi, Nature Photon. 1, 97 (2007).

${ }^{18}$ P. U. Jepsen, D. G. Cooke, and M. Koch, Laser Photonics Rev. 5, 124 (2011).

${ }^{19}$ T. Brabec and F. Krausz, Phys. Rev. Lett. 78, 3282 (1997).

${ }^{20}$ F. Shimizu, Phys. Rev. Lett. 19, 1097 (1967).

${ }^{21}$ R. R. Alfano and S. L. Shapiro, Phys. Rev. Lett. 24, 592 (1970).

${ }^{22}$ R. Huber, F. Tauser, A. Brodschelm, M. Bichler, G. Abstreiter, and A. Leitenstorfer, Nature (London) 414, 286 (2001).

${ }^{23}$ M. Dressel and G. Grüner, Electrodynamics of Solids: Optical Properties of Electrons in Matter (Cambridge University Press, Cambridge, 2002).

${ }^{24}$ N. V. Smith, Phys. Rev. B 64, 155106 (2001).
${ }^{25}$ H. Němec, P. Kužel, and V. Sundström, Phys. Rev. B 79, 115309 (2009).

${ }^{26}$ Here, we can ignore, for simplicity, the dispersion due to a phonon contribution to $\hat{\varepsilon}$, which is generally independent of free carriers.

${ }^{27}$ K. Brennan and K. Hess, Solid-State Electton. 27, 347 (1984).

${ }^{28} \mathrm{~A}$ static Gunn field of GaAs is about $5 \mathrm{kV} / \mathrm{cm}$. A terahertz signal with the central frequency of $1 \mathrm{THz}$ and with the peak $E_{\mathrm{THz}}=$ $100 \mathrm{kV} / \mathrm{cm}$, applied to the $\Gamma$ valley electron, has a ponderomotive energy of about $1.8 \mathrm{eV}$, clearly exceeding the $\Gamma-L$ and $\Gamma-X$ energy separations of 0.33 and $0.52 \mathrm{eV}$, respectively, ${ }^{27}$ thus making intervalley transitions possible.

${ }^{29}$ M. C. Hoffmann and D. Turchinovich, Appl. Phys. Lett. 96, 151110 (2010).

${ }^{30}$ N. A. van Dantzig and P. C. M. Planken, Phys. Rev. B 59, 1586 (1999).

${ }^{31}$ The absorption spectra were calculated based on the Fresnel losses at the air-sample interfaces,$^{18}$ corresponding to the background refractive index $n=\sqrt{\varepsilon_{\mathrm{dc}}}$. The influence of the nonlinear index contribution $\Delta n \ll \sqrt{\varepsilon_{\mathrm{dc}}}$ can be neglected in this case.

${ }^{32}$ X. Liu, J. Lægsgaard, and D. Turchinovich, Opt. Express 18, 15475 (2010).

${ }^{33} \Delta \omega=\int_{\omega} E(\omega) d \omega / \max [E(\omega)]$, where $E(\omega)$ is Fourier amplitude of the corresponding terahertz pulse.

${ }^{34}$ Q. Lin, J. Zheng, J. Dai, I-C. Ho, and X.-C. Zhang, Phys. Rev. A 81, 043821 (2010).

${ }^{35}$ M. van der Poel, J. Mørk, and J. M. Hvam, Opt. Express 13, 8032 (2005).

${ }^{36}$ C. J. Chang-Hasnain and S.-L. Chuang, J. Lightwave Technol. 24, 4642 (2006).

${ }^{37}$ G. P. Agrawal, Nonlinear Fiber Optics (Academic Press, Amsterdam, 2007).

${ }^{38}$ See Supplemental Material at http://link.aps.org/supplemental/ 10.1103/PhysRevB.85.201304 for a movie, showing the evolution of $\mathrm{THz}$ pulses in time domain, and their spectrograms in time- and frequency domain, as the peak THz field changes.

${ }^{39}$ M. Nagai, M. Jewariya, Y. Ichikawa, H. Ohtake, T. Sugiura, Y. Uehara, and K. Tanaka, Opt. Express 17, 11543 (2009).

${ }^{40}$ D. Grischkowsky, S. Keiding, M. van Exter, and Ch. Fattinger, J. Opt. Soc. Am. B 7, 2006 (1990).

${ }^{41}$ J. Pozela and A. Reklaitis, Solid-State Electron. 23, 927 (1980).

${ }^{42}$ The momentum relaxation rate in GaAs at $N \leqslant 10^{17} \mathrm{~cm}^{-3}$ is shown to have a negligible dependency on the driving electric field, ${ }^{41}$ therefore the value of $\tau_{r}=94 \mathrm{fs}$, obtained by linear terahertz spectroscopy, was used throughout this model. 